\title{
LUNG MORPHOMETRY, COLLAGEN AND ELASTIN CONTENT: CHANGES AFTER HYPEROXIC EXPOSURE IN PRETERM RABBITS
}

\author{
Renata Suman Mascaretti, ${ }^{\mathrm{I}}$ Marta Maria Galli Bozzo Mataloun, ${ }^{\mathrm{I}}$ Marisa \\ Dolhnikoff, II Celso Moura Rebello ${ }^{I}$
}

doi: $10.1590 / \mathrm{S} 1807-59322009001100010$

Mascaretti RS, Mataloun MMGB, Dolhnikoff M, Rebello CM. Lung morphometry, collagen and elastin content: changes after hyperoxic exposure in preterm rabbits. Clinics. 2009;64(11):1099-104.

INTRODUCTION: Elastic and collagen fiber deposition increases throughout normal lung development, and this fiber network significantly changes when development of the lung is disturbed. In preterm rats and lambs, prolonged hyperoxic exposure is associated with impaired alveolization and causes significant changes in the deposition and structure of elastic fibers.

OBJECTIVES: To evaluate the effects of hyperoxic exposure on elastic and collagen fiber deposition in the lung interstitial matrix and in alveolarization in preterm rabbits.

METHODS: After c-section, 28-day preterm New-Zealand-White rabbits were randomized into 2 study groups, according to the oxygen exposure, namely: Room air (oxygen $=21 \%$ ) or Oxygen (oxygen $\geq 95 \%$ ). The animals were killed on day 11 and their lungs were analyzed for the alveolar size (Lm), the internal surface area (ISA), the alveoli number, and the density and distribution of collagen and elastic fibers.

RESULTS: An increase in the Lm and a decrease in the alveoli number were observed among rabbits that were exposed to hyperoxia with no differences regarding the ISA. No difference in the density of elastic fibers was observed after oxygen exposure, however there were fewer collagen fibers and an evident disorganization of fiber deposition.

DISCUSSION: This model reproduces anatomo-pathological injuries representing the arrest of normal alveolar development and lung architecture disorganization by just a prolonged exposition to oxygen.

CONCLUSIONS: In the preterm rabbit, prolonged oxygen exposure impaired alveolization and also lowered the proportion of collagen fibers, with an evident fiber network disorganization.

KEYWORDS: Oxygen; Animal model; Lung fibers; Bronchopulmonary dysplasia; Lung injury.

\section{INTRODUCTION}

The histological findings of bronchopulmonary dysplasia (BPD) have been described as simplification, and a widening and reduction of the alveoli number, a decrease of the alveolar surface area, the presence of capillary dimorphism, an increase in alveolar septal thickness, ${ }^{1}$ and a modification

${ }^{\text {I } P e d i a t r i c s ~ D e p a r t m e n t, ~ E x p e r i m e n t a l ~ R e s e a r c h ~ U n i t, ~ F a c u l d a d e ~ d e ~ M e d i c i n a ~}$ da Universidade de São Paulo - São Paulo/SP, Brazil.

II Pathology Department, Faculdade de Medicina da Universidade de São Paulo - São Paulo/SP, Brazil.

Email: renatasmp@uol.com.br

Tel: 55113812.2121

Received for publication on July 13, 2009

Accepted for publication on July 24, 2009

Supported by FAPESP (Grant: 98/10580-2) in the density and disposition of the elastic and collagen fibers. $^{2,3}$

Alveoli formation is a continuous process which starts approximately during the 32nd-to-36th week of gestation and continues until late postnatal life, with progressive transformation of the saccules to alveoli. ${ }^{4}$ The deposition of elastic fibers at the tips of secondary alveolar crests and alveolar mouths suggest that elastin is involved in the formation of new alveoli. ${ }^{4}$ Alterations in the deposition of elastic fibers may be one of the factors that leads to hypoalveolarization. ${ }^{5}$

The etiology of BPD is multi-factorial; clinical and experimental studies ${ }^{6,7}$ suggest that oxygen-related toxicity is one of the most important factors involved. Prolonged hyperoxic exposure is associated with reduction of the 
internal alveolar surface area, impaired alveolarization, ${ }^{8-11}$ and abnormalities in collagen and elastic fiber deposition. ${ }^{12}$ These abnormalities in the development of fibers have not been studied in the preterm rabbit.

Bruce at al. ${ }^{4}$ demonstrated that hyperoxic exposure in the developing rat lung alters the total length and structure of elastic fibers. Parenchymal collagen increases throughout lung development and the presence of a delicate and complex collagen network permits normal septation. In chronic lung disease, development of the lung is disturbed, with thickened collagen around the saccule walls, a widened interstitium, and an increase in quantity and size of interstitial collagen fibers. ${ }^{13}$ Although the preterm rabbit model is used for studies in lung development, ${ }^{14,15}$ the impact of hyperoxic exposure in the preterm rabbit lung has not been evaluated.

A small number of animal models for lung immaturity have been developed using rats, lambs, and baboons. The primate models have been applied for experimental research; ${ }^{10}$ however, there is a need for a complete neonatal intensive care unit with nursing, laboratory and monitoring care, and this is extremely expensive for developing countries.

In 1997, Chen et al., ${ }^{16}$ developed an animal model using premature rats that were subjected to hyperoxia; this is advantageous because of the ease of manipulation and low cost. However, due to the low birth weight of the animals, this model has restrictions in the study of lung mechanics and the alveolar and total lung surfactant pool in premature newborns. Therefore, an animal model of lung immaturity using premature rabbits was developed, which are low cost, easy to handle, and show the arrest of alveolar development and the disorganization of lung architecture.

The objective of the present study was to evaluate the morphological changes in the deposition of elastic and collagen fibers in the lung interstitium, induced by hyperoxic exposure in the preterm rabbit.

\section{MATERIALS AND METHODS}

Animal preparation. The study protocol was approved by the Ethics Committees for the Analysis of Research Projects (CAPPesq), at the Hospital das Clínicas, São Paulo University Medical School. New Zealand-White rabbits were delivered by cesarean section at the $28^{\text {th }}$ day of gestation, and following an adaptation period, the surviving animals were randomized into 2 study groups, as follows: continuous exposition to oxygen $\geq 95 \%$ (Oxygen) or room air (Air). The animals were kept in incubators at $32^{\circ} \mathrm{C}$, and received prophylactic antibiotic therapy from the third day of life ${ }^{15}$ (penicillin crystalline - $20.000 \mathrm{UI} / \mathrm{kg}$ - IM and streptomycin $20 \mathrm{mg} / \mathrm{kg} /$ day - IM) and vitamin $\mathrm{K}(2 \mu \mathrm{g} / \mathrm{kg} /$ day). Feeding was carried out with a milk formula that had a similar composition to natural rabbit milk ${ }^{17}$. The volume of the formula for the first day of life was $100 \mathrm{ml} / \mathrm{kg}, 150 \mathrm{ml} /$ $\mathrm{kg}$ for the second and $200 \mathrm{ml} / \mathrm{kg}$ from the third to eleventh days. Animals were fed twice a day with a $n^{\circ} 4$ orogastric tube. Heated and humidified oxygen was supplied through a sealed acrylic chamber, and the concentration was checked by an environment oxygen analyzer (Dixtal ${ }^{\circledR}$, São Paulo, Brazil). On 11th day after birth, the animals were deeply sedated with sodium pentobarbital (25 mg/kg, IP) and were then killed by sectioning the abdominal aorta.

Preparation and sampling for morphometric and image analysis. The lungs were pressurized with air at $30 \mathrm{~cm} \mathrm{H}_{2} \mathrm{O}$, followed by tracheal ligature. After the lungs were removed from the thorax, they were fixed with a $10 \%$ buffered formol solution for 24 hours, followed by weight determination (TR 403, Denver Instrument Company ${ }^{\circledR}$, United States), and measurement of the total lung volume (TLV) by a water displacement technique adapted from Bruce et al. ${ }^{4,18}$ The lung weight /body weight (g/100g) (LW/ $\mathrm{BW})$ and the lung weight / lung volume (g/ml) (LW/LV) ratios were calculated.

Slide preparation involved $1 \mathrm{~mm}$ thickness sagittal cuts of the distal portion of the right inferior lobe. ${ }^{19}$ The tissue was preserved in $70 \%$ ethylic alcohol, embedded in paraffin, and serial slices of $5 \mu \mathrm{m}$ thickness were obtained. The slides were stained with hematoxylin and eosin for morphometric analysis, with Weigert, modified resorcin-orcein for elastic fiber analysis, and with Picrosirius for collagen analysis.

A Nikon E600 microscope, coupled to a linear pointing count grid (50 lines/100 points) of known area, and an image analysis program (Image-Pro, Media Cybernetics INC ${ }^{\circledR}$, EUA), were used for the morphometric analysis. A minimum of 10 microscope fields was examined for each animal. All of the morphometric and image analysis measurements were performed by the same investigator in a blinded fashion.

Morphometry. The lung developmental process was analyzed by calculating the mean linear intercept $(\mathrm{Lm}),{ }^{20}$ the internal surface area (ISA) ${ }^{21}$ and the alveoli number.

The calculation of the Lm used a grid with 100 points and 50 lines, and the intercept was defined as the crossing of the grid line with the alveolar wall. The Lm was calculated by applying the following formula: $\mathrm{Lm}=$ the total length of the 50 lines / the number of alveoli intercepts $\underline{\underline{2}} \underline{\underline{20}}$

The ISA represents the gas exchange surface, and in turn was calculated according to the following formula ${ }^{21}$ : ISA = 4V/Lm that means: $\mathrm{V}=$ the fixed lung volume at $30 \mathrm{~cm} \mathrm{H}_{2} \mathrm{O}$ transtracheal pressure multiplied by the parenchyma fraction. The parenchymal fraction was calculated with the 100-points and 50-lines grid, where the points that fell over the lung 
tissue were counted, with the exception of points that fell over the blood vessels and bronchi with diameters larger than $2 \mathrm{~mm}$. The number of counting points was divided by 100 .

The total alveoli number was calculated using the modified Dunnil method ${ }^{20}$. Lung cuts stained with hematoxylin-eosin were photographed at a magnification of X100 with an image analyzer (Image-Pro, Media Cybernetics INC ${ }^{\circledast}$, EUA). Three lines were traced at different levels from pleura to pleura, and the intercepting number of alveoli walls that crossed each line was counted, added and then divided by two. The length of the lines was measured and added, and the number of alveoli was divided by this value. The results were expressed by length units (number of alveoli / $\mu \mathrm{m} \mathrm{x} 10^{-3}$ ).

Collagen and elastin content. The terminal airways were also evaluated for the density of the elastic and collagen fibers. The proportion of fibers in the lung parenchyma was determined using the linear point counting grid (magnification X400) in 20 fields per animal. The ratio between the number of points based on the colored fibers and the lung parenchyma was determined.

\section{Statistical analysis.}

An analysis of variance was used to compare the body weight, lung weight, lung weight/ body weight and lung weight / lung volume $(\mathrm{g} / \mathrm{ml})$ ratios, the alveoli number, the $\mathrm{Lm}$ and the ISA. The proportion of collagen and elastic fibers was compared by the Chi-Square test. A $p<0.05$ value was considered to be statistically significant.

\section{RESULTS}

\section{Animals distribution in the study groups}

In the group exposed to oxygen, 17 animals were sacrificed with 11 days $\left(\mathrm{O}_{2}\right.$ Group 11 days), and among the animals exposed to environment air, 19 animals were sacrificed with 11 days (Air Group 11 days). One animal was excluded from the $\mathrm{O}_{2}$ Group due to the considered inadequacy of the material processing technique. Therefore, 18 in the Air Group 11 days and 17 in the $\mathrm{O}_{2}$ Group 11 days were used for the final analysis.

\section{Survival}

We observed a higher survival of the animals when exposed to the environment air (30.8\%), compared to the animals exposed to oxygen (10.8\%). The deaths occurred because of infections (skin, systemic), gastrointestinal perforation, or milk aspiration at the feeding occasion.
Lung weight and volumes. Overall, neither the lung weight nor the lung volume were significantly altered after 11 days of oxygen exposure, although a slight increase was observed in the LW/BW ratio, which was related to a $10 \%$ increase in the lung weight among animals from the Oxygen group. Despite this slight increase in lung weight, the lung volume was reduced by $27 \%$, resulting in a $21 \%$ increase in the LW/LV ratio, secondary to oxygen exposure (Table 1).

Table 1 - Lung weight and volumes

\begin{tabular}{lccc}
\hline & Air $(\mathrm{n}=18)$ & Oxygen $(\mathrm{n}=17)$ & $\mathrm{p}$ \\
\hline Birth weight $(\mathrm{g})$ & $33.1 \pm 5.0$ & $33.2 \pm 4.7$ & 0.95 \\
Body weight at $11 \mathrm{~d}(\mathrm{~g})$ & $40.7 \pm 8,0$ & $41.5 \pm 8.4$ & 0.78 \\
Lung weight $(\mathrm{g})$ & $0.83 \pm 0.24$ & $0.92 \pm 0.14$ & 0.19 \\
$\begin{array}{l}\text { Lung weight /Body weight } \\
\text { (g/100g) }\end{array}$ & $2.07 \pm 0.53$ & $2.26 \pm 0.34$ & 0.22 \\
$\begin{array}{l}\text { Lung volume (ml) } \\
\begin{array}{l}\text { Lung weight / Lung volume } \\
\text { (g/ml) }\end{array}\end{array}$ & $2.63 \pm 1.83$ & $1.93 \pm 0.80$ & 0.16 \\
\hline
\end{tabular}

The Lm, ISA and alveoli number. The Lm increased approximately $40 \%$ among the oxygen exposed animals ( $p$ $<0.05$, Table 2).

Table 2 - Mean linear intercept (Lm), internal surface area (ISA) and alveoli number

\begin{tabular}{lccc}
\hline & Air & Oxygen & $\mathrm{p}$ \\
\hline Lm $(\mu \mathrm{m})$ & $60.5 \pm 18.2$ & $85.3 \pm 27.3$ & $<0.05$ \\
ISA $\left(\mathrm{cm}^{2}\right)$ & $3.3 \pm 1.4$ & $3.1 \pm 1.4$ & 0.68 \\
Alveoli number & $11.5 \pm 2.3$ & $8.5 \pm 2.8$ & $<0.05$ \\
\hline
\end{tabular}

In contrast, although a slight reduction was observed in the Oxygen group regarding the internal surface area after hyperoxic exposure, this difference was not significant (Table 2). In line with the Lm, the alveoli number was reduced by $26 \%$ among the oxygen exposed animals ( $\mathrm{p}$ $<0.05$ Table 2). Hypoalveolarization could be clearly visualized in the animals exposed to oxygen for 11 days, when compared to the Air group (Figure 1).

The collagen and elastin content. The collagen fiber density among the animals from the Oxygen group was 2-fold lower when compared to the animals from the Air group (Table 3).

A disordered deposition of collagen fibers in the lung interstitium was observed (Figure 2). A decrease in the density of elastic fibers was seen in the group exposed to oxygen, although this was not significant (Table 3). In addition, an abnormal deposition of elastin fibers and a 
structural disorganization in the lung parenchyma was also observed in the oxygen exposed animals (Figure 3).

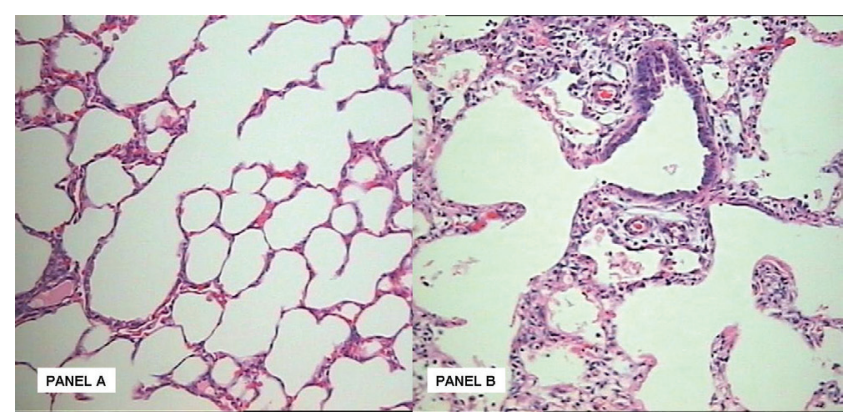

Figure 1 - Panel A:Hematoxilin-eosin stained section (magnification X20) Normal development of the preterm rabbit lung with alveoli of normal size and number. Panel B: After 11 days of hyperoxic challenge there is an evident reduction in alveolar number, with evident accumulation of inflammatory cells in the intersticium.

Table 3 - Collagen and elastin content

\begin{tabular}{lccc}
\hline & Air & Oxygen & $\mathrm{p}$ \\
\hline $\begin{array}{l}\text { Elastic fibers/ } \\
\text { points }\end{array}$ & $0.51 \pm 0.19$ & $0.40 \pm 0.2$ & 0.16 \\
$\begin{array}{l}\text { Collagen fibers/ } \\
\text { points }\end{array}$ & $0.40 \pm 0.18$ & $0.18 \pm 0.08$ & $\mathrm{p}<0.001$ \\
\hline
\end{tabular}

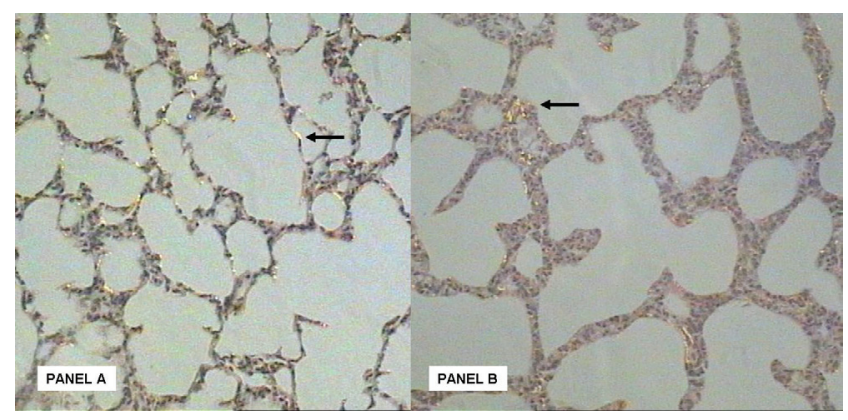

Figure 2 - Panel A: Picrosirius stained section (magnification X20). Collagen fiber deposition in lung parenchyma at the 11th day of life (arrow) at room air. Panel B: After 11 days of hyperoxic challenge there is an evident disordered deposition of the collagen fibers in the lung intersticium (arrow).

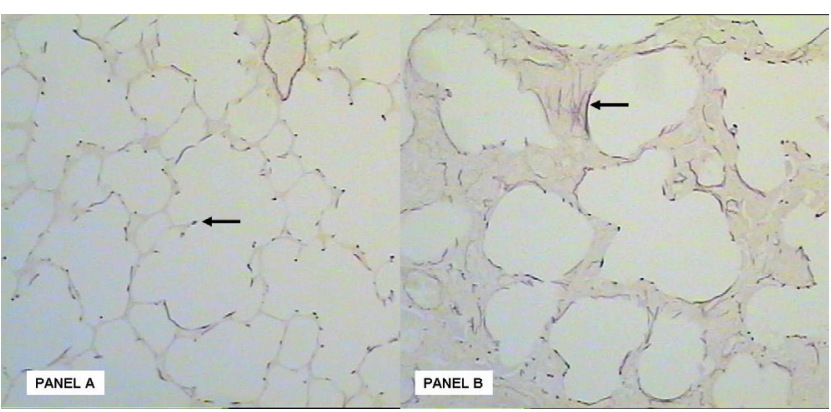

Figure 3 - Panel A: Weigert stained section (magnification X20). Elastin fiber deposition in lung parenchyma at the Air group. There is an organization of elastin fiber in the secondary alveolar septa (arrow). Panel B: After hyperoxic stimuli there is an evident disorganization and abnormal deposition of elastin fibers in the lung intersticium (arrow).
Description of the histology. In the animals exposed to hyperoxia, a reduction in the alveoli number associated with a higher alveoli size, and an increase in the distance between the alveoli, due to septal thickening associated with an intense interstitial fibroproliferation, was observed, as well as a disorganization of the deposition of the elastic and collagen fibers (Figures 1, 2 and 3).

\section{DISCUSSION}

This study investigated, for the first time, the effects of hyperoxia on lung morphometry and the collagen and elastin content in the preterm rabbit model. In rabbits, the gestational period is 31 days, with 28 days of gestation representing the limit of viability, ${ }^{22,23}$ which corresponds to $87 \%$ of the gestation term, and to the saccular phase of lung development. When the alveolarization process starts it would correspond with a premature newborn in the saccular phase of normal lung development. ${ }^{14}$ The association of lung immaturity with hyperoxia was chosen to trigger lung injury, due to the increasing role of free radical participation in the etiopathogeny of the disease.

The pattern of lung injury associated with oxygen exposure is well described in the adult lung, but the effects of oxygen during the phase of growth and development of the lung remain unclear. Nowadays, lung injury is associated to a prolonged exposure to oxygen in an immature lung, and is characterized by a disorganized hypoalveolarization of the lung architecture, an absence of significant alterations of the conducting airways, and variable degrees of fibroproliferation ${ }^{1}$. In our study, using the preterm rabbit, the histopathological analysis demonstrated an arrest of lung development and a disorganization of the lung architecture, associated to the prolonged exposure to hyperoxia.

The total lung volume is composed of a sum of the volumes of the airway spaces, alveoli, capillaries, parenchyma, interstitium, endothelial and epithelial cells $\mathrm{s}^{24}$. After birth, there is a continuous increase in lung volume, which continues throughout aging. ${ }^{25}$ With lung development, there is a redistribution among the compartments which compose the total volume, and this occurs mainly due to an increase in the airway spaces and the capillaries. This parallels a decrease in the septal thickness content, thus enabling gas exchange.

In our study, a trend towards an increase in the LW/ $\mathrm{LV}$ ratio was observed, due to the relative substitution of the airway spaces for abnormal lung parenchyma, and also due to an accumulation of a large amount of cells in the interstitium. The increase in the LW/LV ratio, reflecting lung density, was derived mainly from a $27 \%$ reduction of the $\mathrm{LV}$ after exposure to oxygen. 
During normal lung development, alveolarization results in a decrease in the mean linear intercept and an increase in the internal surface area. In this study, hyperoxia impaired this process, as demonstrated by an increase of the Lm values after oxygen exposure. This observation suggests an increase in alveolar size, secondary to the alveolar septation arrest, and in addition to the increase in distance among the alveoli due to septal thickening, as a result of the intense interstitial fibroproliferation. These findings are similar to the BPD models in premature rats based on hyperoxia, in which there is widening of distal airway spaces, a reduction in the lung volume, and an evolution to fibrosis. ${ }^{16}$

These results associated with the reduction in the number of alveoli observed after oxygen exposure, demonstrate that the lung injury secondary to a hyperoxic environment in the premature rabbit produces morphologic alterations similar to BPD in humans.

Differences between the proportions of collagen and elastic fibers and the lung parenchyma were not observed, but the deposition pattern was altered both in appearance and location. This analysis may have been influenced by the hypercellularity of the lung interstitium of the animals exposed to oxygen, therefore these data may have been underestimated. During the normal developmental process, an increase of collagen deposition in the lung parenchyma is observed. Before 30 weeks of gestation, the collagen interstitial net is fragile, and may also be involved in a prior alveolar septation process..$^{13}$ In lung injury models, the collagen content is altered by its concentration, appearance or deposition. Thibeault et al. ${ }^{13}$ observed an increase in collagen deposition and an arrest in alveolar septation, in relation to controls, among 33 children between 23 and 30 weeks of gestation who were at risk for evolving to BPD, and who died between 5 and 94 days of life.

Regarding the elastic fibers, the oxygen exposed animals had a severe disorganization of elastic deposition, with shorter lengths and a tortuous aspect, similar to that found in the premature lamb mode ${ }^{11}$ and in rats exposed to hyperoxia. ${ }^{4}$ In children at risk for developing BPD, an increase in the lung elastic fiber deposition was proportional to the severity of lung injury after a mixed insult of oxygen and mechanical ventilation. ${ }^{3}$ Albertine et al. (1999) found an increase in the elastic fiber content after a period of 3 to 4 weeks of mechanical ventilation in the premature lamb. ${ }^{2}$ On the other hand, using only hyperoxia in a rat model, a structural disorder with a reduction in the length of the elastic fibers, without differences in concentration, was observed. ${ }^{4}$ We did not find an increase in the proportion of elastic fibers in relation to the lung parenchyma in the animals exposed to high concentrations of oxygen, but their deposition was clearly altered. There are studies that suggest that hyperoxia might be associated with the inhibition of lung elastic content ${ }^{4}$ and the increasing use of mechanical ventilation. $^{11}$

These reports suggest that more studies are needed in order to better clarify the role of collagen and elastin fibers in lung development that are altered by different mechanisms of lung injury.

Until now, only a small number of animal models of lung immaturity had been developed with the lamb, baboon and the Rhesus monkey. From these, the primate models have been applied, almost with exclusivity, for BPD experimental research; ${ }^{10}$ however, these models need an infrastructure of a complete NICU with nursing, laboratory and monitoring care, and this becomes extremely expensive, even for developed countries. A BPD model using premature rats has been recently proposed, and has the advantages of ease of manipulation and low cost. ${ }^{16}$ However, this model also presents restrictions in relation to the lung mechanical study, the alveolar and total lung surfactant pool in premature newborns, due to the low birth weight of the animals. We chose the model of the premature rabbit in this study due to its feasibility of studying lung immaturity. The rabbit has a gestational time that is controlled by programmed couplings, a large number offspring ( 7 to 11 puppies per female), and a large size of newborns when compared to rats. This results in a lower cost of studies of lung mechanics and the surfactant system when compared to bigger species. Here we decided to develop a model for the BPD study using premature rabbits, because of the low cost characteristics, ease in handling, relationship to the immature lung study and similarity with the alterations that were detected in human disease.

Since BPD has a multifactorial pathophysiology, the choice of the animal species and the type of insult causing lung injury becomes essential. In this study, the association between lung immaturity and hyperoxia was chosen as an trigger factor of lung injury due to the increased valorization of the free radicals, which participate in the etiopathogeny of the disease, particularly in the extremely preterm newborn human. The use of developing lungs is relevant because they present a less effective antioxidant system, with altered inflammatory responses, and specific characteristics of the lung injury exist, secondary to volutrauma and barotrauma ${ }^{22}$. Ours results reinforce that the preterm rabbit exposed to prolonged hyperoxia can be used as a model to study anatomo-pathological injuries similar to the BPD, with resulting arrest in growth and normal lung development, allowing studies of lung mechanics and an evaluation of the surfactant system at low costs compared to bigger animals. The proposed model 
may be used for the pathophysiology study, treatment and prevention of the injury associated to the exposition to oxygen in the developing lung.

\section{ACKNOWLEDGMENTS}

The authors thank Dr. Alan Jobe for his critical review.

\section{REFERENCES}

1. Coalson JJ. Phatology of new bronchopulmonary dysplasia. Seminars in Neonatology. 2003;8:73-81.

2. Albertine KH, Jones GP, Starcher BC, Bohnsack JF, Davis PL, Cho SC, et al. Chronic lung injury in preterm lambs - disordered respiratory tract development. Am J Respir Crit Care Med. 1999;159:945-58.

3. Thibeault DW, Mabry SM, Ikechukwu IE, Truog WE. Lung elastic tissue maturation and perturbations during the evolution of chronic lung disease. Pediatrics. 2000;106:1452-9.

4. Bruce MC, Pawlowski R, Tomashefski JF Jr. Changes in lung elastic fiber structure and concentration associated with hyperoxic exposure in the developing rat lung. Am Rev Respir Dis. 1989;140:1067-74.

5. Bruce MC, Schuyler M, Martin RJ, Starcher BC, Tomashefski JF, Wedig $\mathrm{K}$. Risk factors for degradation of lung elastic fibers in the ventilated neonate. Am Rev Respir Dis. 1992;146:204-12.

6. Crapo JD, Barry BE, Foscue HA, Shelburne J. Structural and biochemical changes in rat lungs occurring during exposures to lethal and adaptive doses of oxygen. Am Rev Respir Dis. 1980;122:123-43.

7. Bonikos DS, Bensch KG, Northway WH Jr. Oxygen toxicity in the newborn. The effect of chronic continuous 100 percent oxygen exposure on the lungs of newborn mice. Am J Pathol. 1976;85:623-50.

8. Sobonya RE, Logvinoff MM, Taussig LM, Theriault A. Morphometric analysis of the lung in prolonged bronchopulmonary dysplasia. Pediatr Res. 1982;16:969-72.

9. Coalson JJ, Winter V, Delemos RA. Decreasead alveolarization in baboon survivors with bronchopulmonary dysplasia. Am J Respir Crit Care Med. 1995;152:640-6.

10. Coalson JJ, Kuehl TJ, Escobedo MB, Hilliard JL, Smith F, Meredith K, et al. A baboon model of bronchopulmonary dysplasia. II. pathologic features. Exp Mol Pathol. 1982;37:335-50.

11. Pierce RA, Albertine KH, Starche BC, Bohnsack JF, Carlton DP, Bland RD. Chronic lung injury in preterm lambs: disordered pulmonary elastin deposition. Am J Physiol. 1997;272:452-60.

12. Coalson JJ, Winter V, Siler-Khodr T, Yoder BA. Neonatal chronic lung disease in extremely immature baboons. Am J Crit Care Med. 1999; 160:1333-46
13. Thibeault DW, Mabry SM, Ekekezie I, Zhang X, Truog WE. Collagen scaffolding during development and its deformation with chronic lung disease. Pediatrics. 2003;111:766-76.

14. Karnak I, Muftuoglu S, Cakar N, Tanyel FC. Organ growth and lung maturation in rabbit fetuses. Res Exp Med. (Berl) 1999;198:277-87.

15. Shah M, Bry K, Hallman M. Protective effect of transferrin against hyperoxia: a study on premature rabbits. Pediatr Pulmonol. 1997;24:42937.

16. Chen Y, Martinez MA, Frank L. Prenatal dexametasone administration to premature rats exposed to prolonged hyperoxia: a new rat model of pulmonary fibrosis (bronchopulmonary dysplasia). J Pediatr. 1997;130:409-16.

17. Sogorb F, Damy SB, Rodrigues UP, Chaguri ICAG. Variação na composição do leite de coelha. Mem Inst Butantan. 1991;53:175-80.

18. Scherle, W. A simple method for volumetry of organs in quantitative stereology. Mikroskopie. 1970;26:57-61.

19. Kikkawa Y, Kaibara M, Motoyama EK, Orzalesi MM, Cook CD. Morphologic development of fetal rabbit lung and its acceleration with cortisol. Am J Pathol. 1971;64:423-42.

20. Dunnil MS. Quantitative methods in the study of pulmonary pathology. Thorax. 1962;17:320-8

21. Frank L. Oxygen toxicity in neonatal rats: the effect of endotoxin treatment on survival during and post- $\mathrm{O}_{2}$ exposure. Pediatr Res. $1987 ; 21: 109-15$

22. Lorenzo AV. The preterm rabbit: a model for the study of acute and chronic effects of premature birth. Pediatr Res. 1985;19:201-5.

23. Rossi FS, Mascaretti RS, Haddad LB, Freddi NA, Mauad T, Rebello $\mathrm{CM}$. Utilization of the lower inflection point of the pressure-volume curve results in protective conventional ventilation comparable to high frequency oscillatory ventilation in an animal model of acute respiratory distress syndrome. Clinics. 2008;63:237-44.

24. Burri PH. The postnatal growth of the rat lung III. Morphology. Anat Rec. 1974;180:77-98.

25. Zeltner TB, Caduff JH, Gehr P, Pfenninger J, Burri PH. The postnatal development and growth of the human lung. II. Morphometry. Respir Physiol. 1987;67:247-67. 